\title{
Sparse and Dense Mixed Grid Transit Accessible Network Based on Uneven Distribution of Travel Demand
}

\author{
Chen Guo $\mathbb{D}^{1},{ }^{1,2}$ Jianjun Wang $\mathbb{D}^{1},{ }^{1}$ Yueying Huo $\mathbb{D}^{2,3}$ and Meiying Jian ${ }^{2}$ \\ ${ }^{1}$ College of Transportation Engineering, Chang'an University, Xi'an 710064, China \\ ${ }^{2}$ Transportation Institute, Inner Mongolia University, Huhhot 010070, China \\ ${ }^{3}$ School of Mathematical Science, Inner Mongolia University, Huhhot 010021, China \\ Correspondence should be addressed to Jianjun Wang; wjjun16@chd.edu.cn
}

Received 8 December 2020; Revised 22 January 2021; Accepted 2 February 2021; Published 18 February 2021

Academic Editor: Tingsong Wang

Copyright (C) 2021 Chen Guo et al. This is an open access article distributed under the Creative Commons Attribution License, which permits unrestricted use, distribution, and reproduction in any medium, provided the original work is properly cited.

The uneven distribution of travel demand is incredibly commonplace in cities, but insufficient attention has been paid to this problem. In this paper, we explore the impact of the uneven distribution of travel demand on an accessible network. A model with a sparse and dense mixed grid transit network based on an uneven distribution of travel demand is proposed to provide a highperformance bus service. The transit network was composed of two parts: a dense grid network in the downtown area and a sparse grid network in the periphery. The objective function of the model included agency cost and passenger cost, where the decision variables were the downtown-to-city ratio, the downtown headway, stop spacing (line spacing), and ratio of the periphery headway to the downtown headway. This study validated the proposed model using the demands of San Francisco. The concentrated spatial demand resulted in a lower total cost, whereas the varying travel demand must be controlled within an appropriate range to maintain the bus performance. The stable bus lines and stops with a variable timetable of the proposed model are profitable for fast-growing cities.

\section{Introduction}

Public transportation is a key way to solve the low efficiency of traffic and serious environmental pollution caused by the proliferation of private vehicles. Increasing the competitiveness of the bus systems reduces private car travel. To attract more residents to travel by bus, transit agencies must provide a high-quality level of service. A bus network having good coverage in time and space is one of the main ways to improve the level of service for buses. Bus services with good coverage in time and space include the following elements: high spatial coverage of stops, accessible lines for passengers between any origin-destination (OD) pairs, high frequency of vehicles, and long service time [1]. A simple and robust bus network is helpful for optimizing these elements.

The bus network planning method can be divided into two categories: direct networks and accessible networks [1]. The direct network [2-11] aims to reduce passenger transfers by designing multidirectional lines based on the OD matrix.
However, the following problems exist for direct networks: (1) The cost of bus agencies is high because of the high duplication of bus lines and the complexity of the network. (2) The frequency and service time of different lines are greatly affected by the OD matrix. Therefore, it is inconvenient for passengers to travel when there is less demand in the OD matrix. (3) With the development of the city, the bus lines need to be completely replanned, which would cause the bus system to have poor stability [12-17].

To overcome the above problems in direct networks, some researchers have developed an accessible network that minimizes the door-to-door travel time of passengers, although the transfers will increase. For an accessible network, the plans of the network are not based on the OD matrix. The design of the bus network is based on the geometry of the urban road structures and travel demand functions, such as direct-service everywhere [18], radial networks [19], grid networks [20], radial-ring networks [21], and mixed networks [12-17]. Compared with the direct network, the 
accessible network has the following advantages: (1) The design of bus lines in the network is based on simplifications and reduces the duplication of the bus lines, which increases the frequency of all bus lines and is easy to understand. (2) An accessible network is human-oriented to passengers with flexible demands [22]. In the above studies, the grid-radial hybrid network with uniform demand proposed by Daganzo [12] is notable. The grid-radial hybrid network can substantially reduce the travel time of passengers without increasing the cost of bus agencies. Estrada et al. applied the results of Daganzo [12] to redesign the transit network of Barcelona [13]. By comparing the network of Barcelona before and after the transformation, it was proven that the mode share of transit greatly increased after the transformation [14]. Hugo et al. [15], Nocera et al. [16], and Chen et al. [17] transformed the network by Daganzo [12] for cities with different structures and travel demand functions.

In practice, travel demand is more concentrated in downtown areas, whereas, in the periphery, travel demand decreases. In other words, travel demand is always uneven in cities. The problem of uneven distribution of travel demand is more complex, but it is more accurate. Most current studies do not consider uneven travel demand when investigating the issue of accessible networks. In this paper, the impact of uneven travel demand on an accessible network is discussed. The grid-radial hybrid network was composed of a grid network in the downtown area and a radial network in the periphery, so the network would be less stable because of the development of the downtown area. Therefore, a sparse and dense mixed grid transit network is proposed, which transforms the grid-radial hybrid network to improve stability. The main purpose of this study is to provide a feasible method for bus line design and frequency allocation in transit planning. This is crucial for enhancing public transportation performance.

The structure of the paper is as follows: part 2 is the section introducing the sparse and dense mixed grid transit network, along with the objective function, constraints, and decision variables of the model; the results are presented in part 3 to validate the proposed model; part 4 analyzes the changes of spatial demands that were conducted, and the sparse and dense mixed grid transit network is compared with the grid-radial hybrid network; finally, part 5 presents the conclusions and considerations for future work.

\section{Materials and Methods}

2.1. Sparse and Dense Mixed Grid Network. The sparse and dense mixed grid network is used in cities with grid road networks and downtown areas at corners, such as San Francisco, Shanghai, Shantou, and Hohhot in China. The two adjacent cities of the downtown areas are close to the sides of the city. Cities are always limited by natural environmental factors or are developed according to their economy. The uneven distribution of urban structures has bigger impact on the transit planning. These types of sites are shown in Figure 1. The travel demand is unevenly distributed in space and time. The downtown demand is evenly distributed, and the peripheral travel demand decreases linearly with the distance from the edge of downtown. The travel demand downtown is $f(f \geq 1)$ times that at the edge of the periphery. Let the downtown travel demand density function be 1 , and let the periphery demand function be $(1-k x)$, where $x$ is the distance from the travel origin to the edge of the downtown area in the periphery and $k$ is a function of $f: k=((1-(1 / f)) /((1-\alpha) D))$. Passenger travel in a city is time-independent. The number of passengers traveling is $\Lambda$ (pax/h) during peak hours and averages $\lambda$ ( $\mathrm{pax} / \mathrm{h}$ ) hourly during service time. $\lambda$ is a constant.

According to the distribution of passenger demand, the layout of the bus network is a dense grid in the downtown area and a sparse grid in the periphery. The bus lines were designed in two directions. The model included four decision variables: spatial variables and time-related variables. Spatial variables are the bus stop spacing $s(\mathrm{~km})$ and $\alpha$, which is the ratio of the downtown side length $d(\mathrm{~km})$ to the city side length $D(\mathrm{~km})$. The headway downtown $H(\mathrm{~h})$ and the ratio of headway in the periphery to the headway downtown are time-related variables. The bus stop spacing in the entire city is $s$. The headway downtown is $H$; in the periphery, one vehicle runs on $n$ roads and the headway is $n H$. The spatial coverage of the transit is constant throughout the city, whereas the time coverage in the periphery is less than that in downtown areas. Compared to the even grid network, the distance traveled by vehicles and the number of vehicles are reduced, and the bus service maintains a high time and spatial coverage. For passengers, the maximum transfer is one, and the network is more convenient to read and remember.

2.2. Agency Cost. The objective function of the proposed model is to minimize the total cost of the network. To achieve this, agency and passenger costs are considered. The agency cost is composed of the lane construction cost $L$ $(\mathrm{km})$, cost of the total distance traveled by vehicles per hour $V$ (veh.km/h), and cost of the total number of vehicles per hour $M(\mathrm{veh} / \mathrm{h})$. The lane construction cost is the fixed capital investment, whereas the others are flexible and belong to the operating costs. If bus lanes exist, the lane construction cost is zero. $M$ is the ratio of $V$ to the travel speed $v_{c}(\mathrm{~km} / \mathrm{h})[12]$. The full derivations of these parameters are presented in Appendix.

$$
\begin{aligned}
L & =\frac{2 D^{2}}{s}, \\
V & =\frac{4 D^{2}\left(\alpha^{2} n-\alpha^{2}+1\right)}{H n s}, \\
M & =\frac{V}{v_{c}} .
\end{aligned}
$$

2.3. Passenger Cost. The passenger cost includes the access and egress time $A(\mathrm{~h})$, waiting time $W(\mathrm{~h})$, in-vehicle time $T$ (h), and transfer time. In-vehicle time is the ratio of the expected travel distance $E(\mathrm{~km})$ to $v_{c}$. The transfer time is 

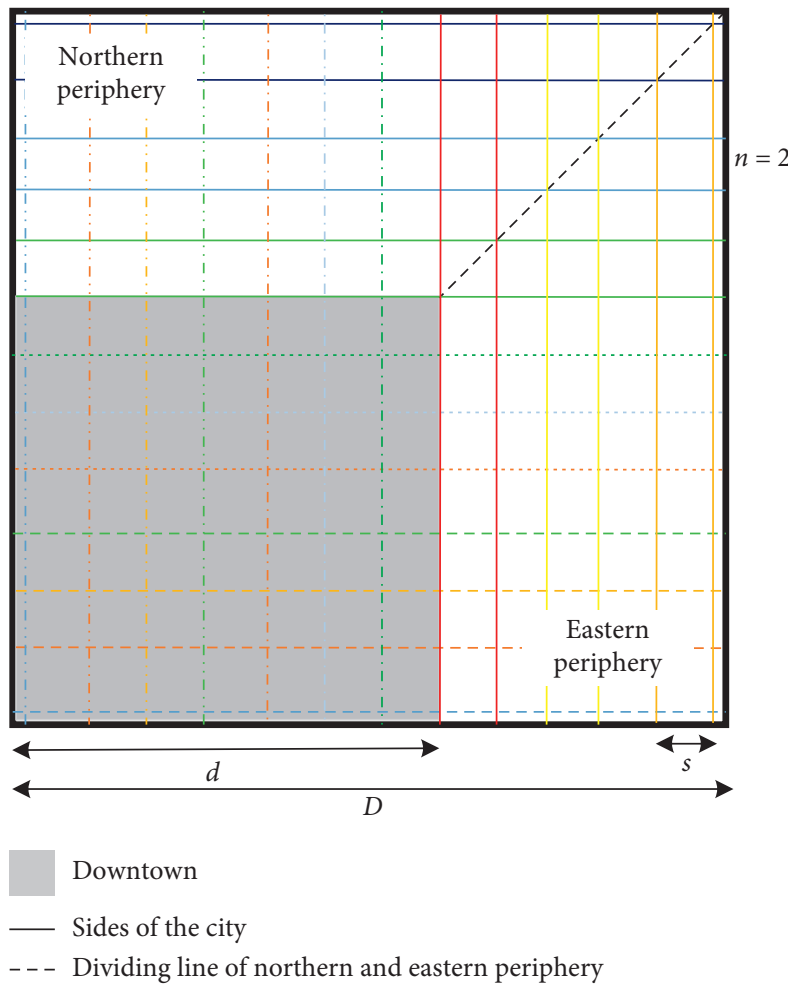

Figure 1: The sparse and dense mixed grid model. ${ }^{*}$ Lines of the same line type and color are run by the same fleet.

calculated by the expected number of transfers per trip $e_{T}$, average transfer distance $b(\mathrm{~km})$, and walking speed $v_{w}$ $(\mathrm{km} / \mathrm{h})$ [23]. The maximum occupancy in critical lines during peak hours $O(\mathrm{pax} / \mathrm{h})$ is also a key parameter to determine whether the bus capacity $C(\mathrm{pax} / \mathrm{h})$ can meet the travel demand [24]. Passengers are distributed based on an all-or-nothing basis. The assumptions are as follows: (1) passengers choose bus lines according to the minimum time, and (2) they choose the nearest bus stop. If two bus stops can be used and they are equidistant to the passenger, then they randomly choose a bus stop. According to this assumption, equations (2)-(8) are the expressions of the parameters related to passenger cost. Appendix includes the calculation procedure for each parameter.

$$
\begin{aligned}
& v_{c}=\frac{1}{\left((1 / v)+(\tau / s)+\Lambda \tau^{\prime}\left(1+e_{T}\right) / V\right)}, \\
& e_{T}=1-\frac{9 \alpha^{2} f s(D+\alpha D+f D-2 f s-\alpha f D)-3 s\left(D-s+\alpha D+f D-f s-\alpha^{2} f D\right)\left(2 \alpha+f-2 \alpha f-5 \alpha^{2} f+2 \alpha^{2}+2\right)}{2 D^{2}\left(2 \alpha+f-2 \alpha f-2 \alpha^{2} f+2 \alpha^{2}+2\right)^{2}},
\end{aligned}
$$

$$
A=\frac{s}{v_{w}}
$$

$$
\begin{aligned}
W= & \frac{H\left(2 n+2 \alpha n+f n+3 \alpha^{2} f+2 \alpha^{2} n-2 \alpha f n-5 \alpha^{2} f n\right)}{2\left(2 \alpha+f-2 \alpha f-2 \alpha^{2} f+2 \alpha^{2}+2\right)} \\
& \times\left[2-\frac{3 s\left(2 \alpha+f-2 \alpha f+5 \alpha^{2} f+2 \alpha^{2}+2\right)(D-s+\alpha D+f D-f s-\alpha f D)}{2 D^{2}\left(2 \alpha+f-2 \alpha f-2 \alpha^{2} f+2 \alpha^{2}+2\right)^{2}}-\frac{9 \alpha^{2} f(D+\alpha D+f D-2 f s-\alpha f D)}{2 D^{2}\left(2 \alpha+f-2 \alpha f-2 \alpha^{2} f+2 \alpha^{2}+2\right)^{2}}\right]
\end{aligned}
$$




$$
\begin{aligned}
E= & \frac{3 D\left(50 \alpha^{5} f^{2}-100 \alpha^{5} f+50 \alpha^{5}-124 \alpha^{4} f^{2}+24 \alpha^{4} f+100 \alpha^{4}+129 \alpha^{3} f^{2}-167 \alpha^{3} f+38 \alpha^{3}-3 \alpha^{2} f^{2}-183 \alpha^{2} f\right)}{70\left(2 \alpha+f-2 \alpha f-2 \alpha^{2} f+2 \alpha^{2}+2\right)^{2}} \\
& +\frac{3 D\left(186 \alpha^{2}-65 \alpha f^{2}-59 \alpha f+124 \alpha+13 f^{2}+65 f+62\right)}{70\left(2 \alpha+f-2 \alpha f-2 \alpha^{2} f+2 \alpha^{2}+2\right)^{2}}, \\
T= & \frac{E}{v_{c}}, \\
O= & \frac{2 s H \Lambda}{\alpha D}\left(\frac{1}{2}-\frac{\alpha^{4}(2 \alpha+3 f-5 \alpha f)^{2}}{8(1-\alpha)^{2}\left(2 \alpha+f-2 \alpha f-2 \alpha^{2} f+2 \alpha^{2}+2\right)^{2}}\right),
\end{aligned}
$$

where $v(\mathrm{~km} / \mathrm{h})$ denotes the cruising speed, which is the same as private cars and is affected by traffic management and control measures; $\tau$ (h/stop) denotes the bus dwelling time at each stop; and $\tau^{\prime}(\mathrm{h} / p)$ denotes the average passengers' boarding time.

2.4. Objective Function. The objective function $Z$ of the proposed model minimizes the total cost, which includes the cost of the passenger and the agent. Agency cost considers the fare of travel, and passenger cost considers the travel time. This study converts agency cost to the time of each passenger [12]. The three parts of agency costs are multiplied by the weight variables $\pi_{V}=\left(c_{V} /(\lambda \mu)\right), \pi_{M}=\left(c_{M} /(\lambda \mu)\right)$, and $\pi_{L}=\left(c_{L} /(\lambda \mu)\right)$, where $\mu$ is the time value of passengers. The four components of passenger costs are multiplied by weight variables, namely, $w_{A}, w_{w}, w_{T}$, and $w_{e}$ [24]. The objective function and constraints are given in the two following equations, respectively:

$\min Z=\left[\pi_{V} V+\pi_{L} L+\pi_{M} M\right]+\left[w_{A} A+w_{w} W+w_{T} T+w_{e} \frac{b}{v_{w}} e_{T}\right]$,

$$
\text { s.t. } s>0 ; H_{\min } \leq H \leq H_{\max } ; \frac{s}{D \leq \alpha \leq 1} ; O \leq C ; M \in N^{*} \text {. }
$$

The first bracket of the objective function is the agency $\operatorname{cost} Z_{A}$, and the second is the passenger cost $Z_{U}$. The second constraint limits the minimum and maximum headway values. $H_{\min }$ denotes the minimum headway to avoid bunching [25], and $H_{\max }$ denotes the maximum headway to maintain the time coverage. The fourth constraint restricts the maximum occupancy $O$ in vehicles and does not exceed the capacity of the vehicles $C$.

2.5. Results. This paper presents a numerical method to measure the effect of the proposed network. The city size and travel demand, in this case, are consistent with those of San Francisco [26, 27], in which $\mu$ is approximately $\$ 20 / \mathrm{h}[12]$. To achieve this, bus rapid transit (BRT) is the most adequate mode [12]. Therefore, BRT was chosen as the basic mode in this study. The values of parameters were taken from the literature originating from the United States, Europe, and China [12-17, 25, 28-31]. In those studies, factors such as the type of bus lanes, signal delays, bus bunching, and criteria of highquality service were considered. The access and egress time, waiting time, and transfer time for passengers may cause discomfort and anxiety. Therefore, $w_{A}, w_{w}$, and $w_{e}$ were set to $2.2,2.1$, and 2.5 , respectively [24]. The weight of the in-vehicle time was 1 . The walking speed of pedestrians was approximately $4.5 \mathrm{~km} / \mathrm{h}$, whereas $v_{w}$ was $2 \mathrm{~km} / \mathrm{h}$ owing to delays caused by signal control and detours. For ease of calculation, $f$ was set to 1 . The parameters used are listed in Table 1. The model can produce the global optimal solution using the Lingo software.

It took 37 minutes to obtain the global optimal solution, and the results are listed in Table 2. The solution time of the model was reasonable. Because $f$ was 1 , the travel demand was evenly distributed throughout the city. $\alpha$ was close to 1 . The ratio of the downtown area to the entire city area was approximately 0.92 . The headway was about four minutes in the downtown area and $n$ was equal to 1 , so the headway in the periphery was also approximately four minutes. The stop spacing was approximately $406 \mathrm{~m}$. For the entire city, the space-time coverage was consistent. The total cost of each passenger was $1.22 \mathrm{~h}$. The average passenger cost was $0.96 \mathrm{~h}$, and the average passenger travel time $Z_{U T}$ was $0.62 \mathrm{~h}$. The agency cost for each passenger was $0.26 \mathrm{~h}$. The maximum occupancy in critical lines during peak hours had not reached the capacity of the vehicle, so the passenger travels during peak hours could increase. The total costs were stable and less affected by the decision variables, according to the sensitivity analysis. This is similar to the results obtained by Daganzo [12] and Chen [17].

\section{Discussion}

3.1. Spatial Travel Distribution. The distribution of spatial demand has a greater impact on travel time and bus costs, and it is closely related to urban planning and land use. In 
TABLE 1: Value of model parameters.

\begin{tabular}{|c|c|c|c|c|c|c|c|c|c|c|}
\hline $\begin{array}{l}\text { Parameter } \\
\text { Value }\end{array}$ & $\begin{array}{c}D(\mathrm{~km}) \\
10\end{array}$ & $\begin{array}{c}\lambda(\mathrm{p}) \\
20,000\end{array}$ & $\begin{array}{c}\wedge(\mathrm{p}) \\
50,000\end{array}$ & $\begin{array}{c}\mu(\$ / \mathrm{h}) \\
20\end{array}$ & $\begin{array}{c}c_{L}(\$ / \mathrm{km}) \\
90\end{array}$ & $\begin{array}{c}c_{V}(\$ / \mathrm{veh} \cdot \mathrm{km}) \\
2\end{array}$ & $\begin{array}{c}c_{M}(\$ / \text { veh }) \\
40\end{array}$ & & $v_{A}$ & $\begin{array}{l}w_{w} \\
2.1\end{array}$ \\
\hline Parameter & $w_{e}$ & $w_{T}$ & $v_{w}(\mathrm{~km} / \mathrm{h})$ & $C$ (p/veh) & $\tau(h /$ stop $)$ & $\tau^{\prime}(\mathrm{h} / \mathrm{p})$ & $v(\mathrm{~km} / \mathrm{h})$ & $b(\mathrm{~km})$ & $H_{\min }(\mathrm{h})$ & $H_{\max }(\mathrm{h})$ \\
\hline
\end{tabular}

TABLe 2: Model results.

\begin{tabular}{lccccccccc}
\hline Parameter & $\alpha$ & $H(\min )$ & $s(\mathrm{~m})$ & $n$ & $L(\mathrm{~km})$ & $M(\mathrm{veh})$ & $V(\mathrm{veh} . \mathrm{km} / \mathrm{h})$ & $v_{c}(\mathrm{~km} / \mathrm{h})$ \\
Value & 0.96 & 4.0 & 406 & 1 & 492.82 & 759 & 14947.51 & 19.69 \\
\hline Parameter & $A(\mathrm{~h})$ & $W(\mathrm{~h})$ & $T(\mathrm{~h})$ & $e_{T}$ & $Z_{U}(\mathrm{~h})$ & $Z_{U T}(\mathrm{~h})$ & $Z_{A}(\mathrm{~h})$ & $Z(\mathrm{~h})$ & $O(\mathrm{p})$ \\
Value & 0.20 & 0.06 & 0.34 & 0.96 & 0.96 & 0.62 & 0.26 & 1.22 & 138 \\
\hline
\end{tabular}

this section, changes in the spatial demand are discussed. $f$ changed from 1 to 1.9 , whereas the other parameters remained unchanged. The larger the value of $f$ was, the more concentrated the travels were downtown.

The changes in the objective function and the decision variables with spatial demand are shown in Figures 2 and 3. According to the results, when the travel demand was more concentrated in the downtown area, $Z$ and $Z_{U}$ significantly dropped, whereas $Z_{A}$ slowly increased. For agents, resources were more concentrated in downtown areas to provide better service, where the demand was higher, and the average travel distance and time were reduced for passengers. The growth in agency costs was smaller than the decline in average travel time. Therefore, the total cost was reduced. When $f$ increased, $\alpha$ and $s$ decreased. $H$ was between 4 and 5 min. $n$ varied from 1 to 4 and was only one nonmonotonic decision variable. The stop spacing was changed from 406 to $309 \mathrm{~m}$. In this case, the spatial coverage of the network increased. The ratio of the downtown area to that of the whole city and stop spacing were relatively stable, whereas the headway was more variable. As a result, the lines and stops of the network were stable and the timetable was flexible. Above all, the bus system is relatively stable in the proposed model.

Although the speed of vehicles will decrease with more concentrated demands, passengers still prefer to live in cities with dense demands. When $f$ was 1.4 , the total cost and maximum occupancy in the critical line during peak hours were minimal. When $f>1.9$, the model had no solution, and the downtown area drastically changed. Therefore, based on the goals of reducing passenger travel time and maintaining the competitiveness of buses, the spatial density difference in cities should be bounded.

3.2. Comparison between Sparse and Dense Mixed Grid and Grid-Radial Hybrid Networks. In this section, the sparse and dense mixed grid network and the grid-radial hybrid net- work are compared. The hybrid network was composed of a grid network in the downtown area and a radial network in the northern and eastern periphery [17]. The two types of networks were compared when $f$ was 1 and 1.6. When $f>1.6$, the hybrid network collapsed [17]. The other parameters were the same as before.

As shown in Table 3, for the decision variables, the values for $s$ in the two types of networks are close. However, the values for $\alpha$ significantly change from one network to another. The ratio of the downtown area to the city area in the mixed grid network was always larger than that in the hybrid network. The values for $H$ are similar, and the headway in the grid network is always larger than that in the hybrid network.

For the objective functions, when $f$ was 1 , the total cost of the hybrid network was lower for the agent and passengers. In the grid network, the number of transfers was smaller and the travel speed was faster. When $f$ was equal to 1.6 , the grid network performed better. Although the agency cost increased, passenger costs decreased considerably. The total cost of the grid network decreased by $34 \%$ compared to that of the hybrid network. The agency cost was similar, whereas the passenger cost in the grid network was much lower. The significant decrease in in-vehicle time was a result of the simpler bus network. The average in-vehicle time was smaller in the grid network, which is more attractive to passengers. Under different circumstances, the length of bus lines in the periphery was longer in the grid network, but most cities do not need bus lane construction costs owing to the existing lanes. The greater the difference in space demand is, the more noticeable the advantages of the mixed grid network are.

Network stability was also compared. For the bus system, the bus lines and stops were relatively stable, whereas the timetable varied more. With the development of cities, the headway in the periphery of the grid network can only be reduced. Naturally, the stable bus lines and stops are much 


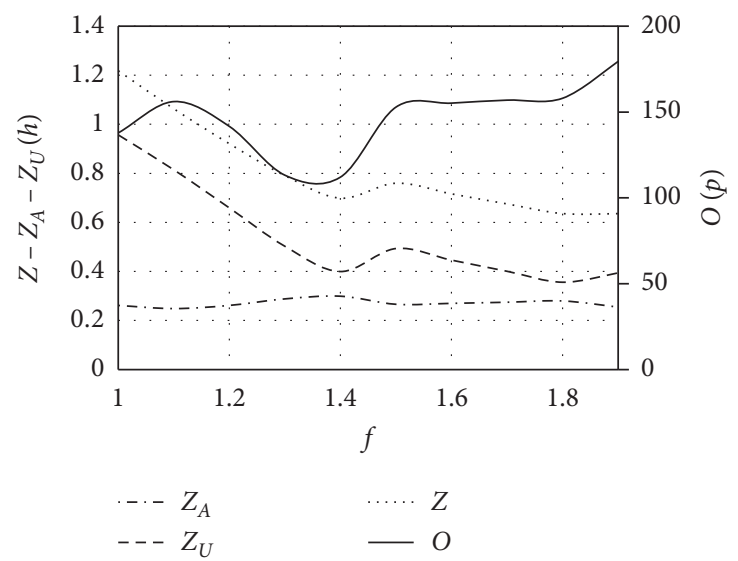

Figure 2: Change of the objective function with $f$.

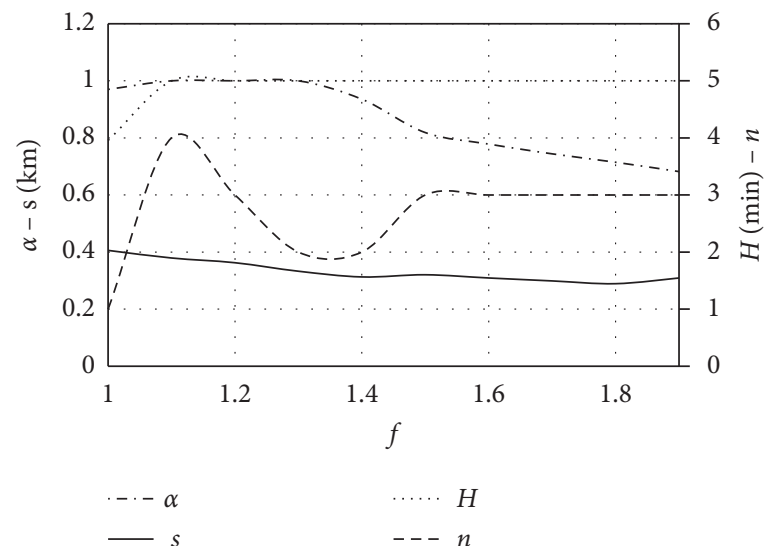

Figure 3: Change of the decision variables with $f$.

TABLE 3: Contrast of the sparse and dense mixed grid network and the grid-radial hybrid network.

\begin{tabular}{cccccccccc}
\hline & & $\alpha$ & $H(\mathrm{~min})$ & $s(\mathrm{~m})$ & $n$ & $V(\mathrm{veh} \cdot \mathrm{km} / \mathrm{h})$ & $M(\mathrm{veh})$ & $L(\mathrm{~km})$ & $v_{c}(\mathrm{~km} / \mathrm{h})$ \\
\hline \multirow{2}{*}{$f=1$} & Hybrid & 0.78 & 3 & 378 & - & 12638.61 & 669.00 & 426.44 & 18.89 \\
\multirow{5}{*}{$f=1.6$} & Grid & 0.97 & 4 & 406 & 1 & 14947.51 & 759.00 & 492.82 & 19.69 \\
& Hybrid & 0.68 & 3 & 334 & - & 11058.20 & 625.00 & 438.52 & 17.69 \\
& Grid & 0.78 & 5 & 309 & 3 & 11443.97 & 673.00 & 646.76 & 17.00 \\
\hline \multirow{2}{*}{$f=1$} & & $A(\mathrm{~h})$ & $W(\mathrm{~h})$ & $e_{T}(\mathrm{~h})$ & $T(\mathrm{~h})$ & $Z_{U}(\mathrm{~h})$ & $Z_{A}(\mathrm{~h})$ & $Z(\mathrm{~h})$ & $O(\mathrm{pax} / \mathrm{h})$ \\
\hline \multirow{4}{*}{$f=1.6$} & Hybrid & 0.19 & 0.05 & 1.01 & 0.34 & 0.91 & 0.23 & 1.14 & 76.00 \\
& Grid & 0.20 & 0.06 & 0.96 & 0.34 & 0.96 & 0.26 & 1.22 & 138.00 \\
& Hybrid & 0.15 & 0.05 & 0.91 & 0.23 & 0.74 & 0.22 & 0.96 & 33.00 \\
& Grid & 0.17 & 0.06 & 0.95 & 0.10 & 0.45 & 0.27 & 0.72 & 155.00 \\
\hline
\end{tabular}

more attractive to passengers with flexible travel demands. Therefore, the mixed grid network is more meaningful for developing cities because of the changes in urban planning and land use.

\section{Conclusions}

This paper proposes a sparse and dense mixed grid network based on the uneven travel demand facing cities with downtown areas at corners. The proposed model was tested by a numerical method in which the travel demand and urban structure are consistent with those in San Francisco. The global optimal solution was obtained within a satisfactory time using Lingo software. The impacts of uneven travel on an accessible network, such as bus line and frequency, are discussed. At present, few studies have focused on accessible bus networks, and the impacts of uneven travel demand on accessible networks have often been ignored. 


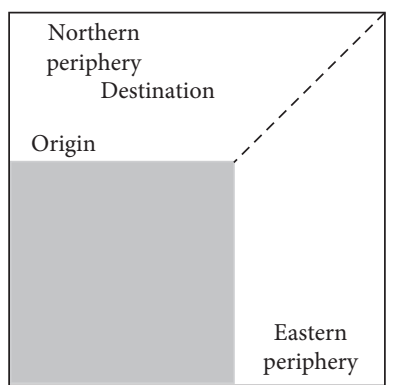

(a)

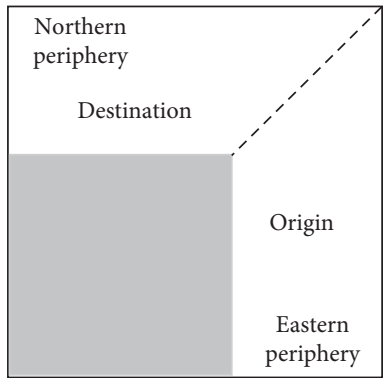

(d)

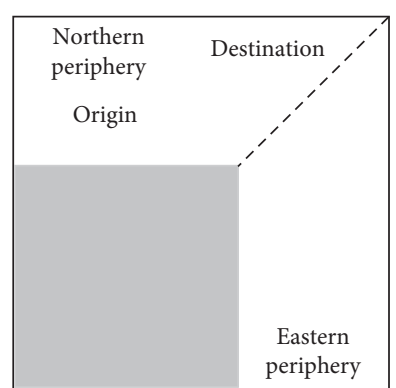

(b)

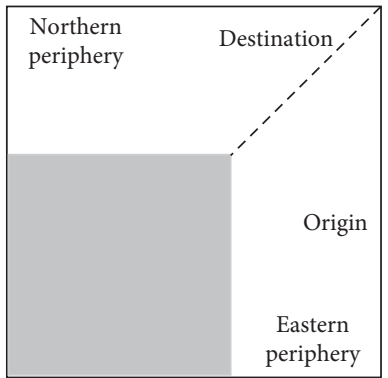

(e)

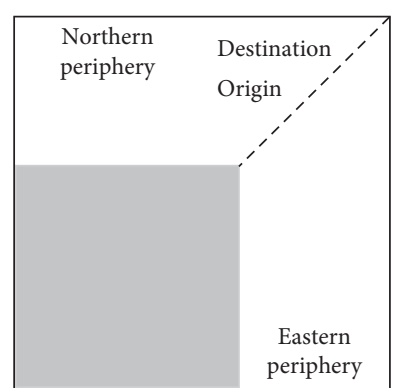

(c)

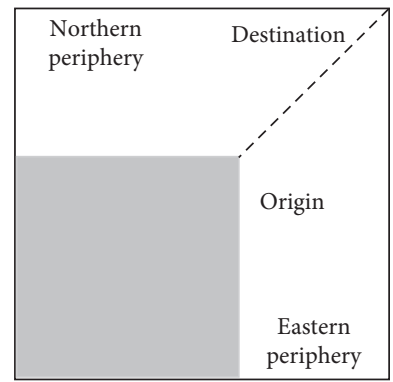

(f)

Figure 4: Different situations for case (b).

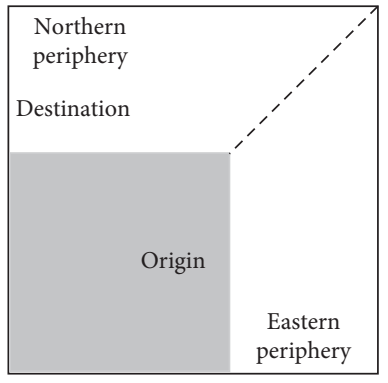

(a)

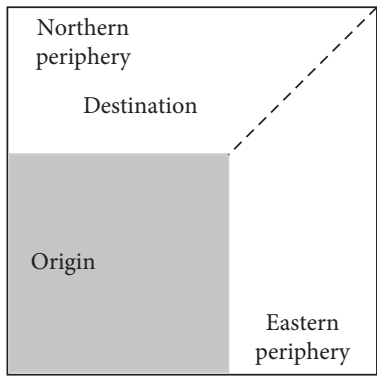

(b)

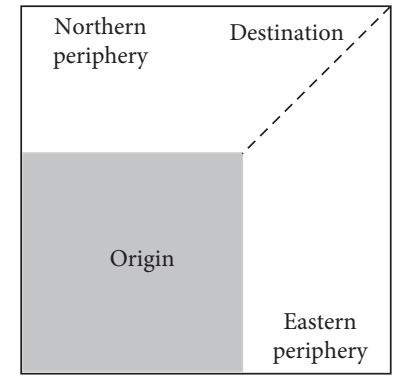

(c)

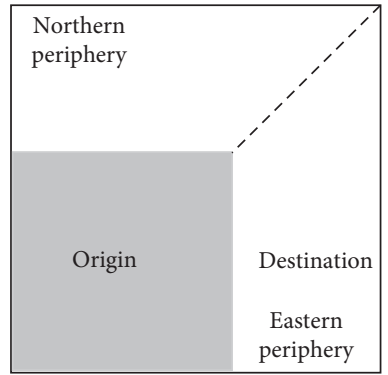

(d)

Figure 5: Four situations for case (c).

This type of network can be conducive to improving the stability of lines and stops and decreasing the in-vehicle time of passengers when compared with the grid-radial hybrid network. A sparse and dense mixed grid network is more beneficial for developing cities.

Analyses of the changes in spatial travel distribution were conducted. The results show that the model was consistent with real bus networks. Differences in spatial demand are critical for the sustainability and performance of transit. The concentrated spatial demand results in a lower total cost and better usage of buses by the public. Cities with concentrated demands are preferred by passengers because those cities have less access and egress time. However, if excessive travel is concentrated in downtown areas, it is not convenient for passengers in the periphery. As a result, bus competitiveness is weakened. Differences in land use should be planned within a reasonable range. The sparse and dense mixed grid network and grid-radial hybrid network were compared. The greater the difference in spatial demand is, the more noticeable the advantages of the sparse and dense mixed grid network are. The simple lines reduce the invehicle time of passengers and total costs. The stable bus lines and stops and the variable timetable of the sparse and dense mixed grid transit network are beneficial for fastgrowing cities.

The bus design for cities with neighboring satellite cities, such as New York, Los Angeles, and Detroit, should be one of the focus points in future studies. The design problem of 
clean energy buses, such as fleet size, lines, and stop locations, should also be considered, especially under stochastic charging demands [32].

\section{Appendix}

Result 1. The total length of the two-direction bus lane is given by $L=\left(2 D^{2} / s\right)$.

Proof. Stops receiving double spatial coverage were associated with $2 s$. The number of stops was $\left[D^{2} / s^{2}\right]$.

Result 2. The total distance traveled by vehicles per hour is given by $V=\left(\left(4 D^{2}\left(\alpha^{2} n-\alpha^{2}+1\right)\right) / H n s\right)$.
The distance traveled by the vehicles is the ratio of the line length to the headway. The length of distance traveled is twice the length of the lines downtown: $2\left[2\left(D^{2} / s\right) \alpha^{2}\right]=\left(\left(4 D^{2} \alpha^{2}\right) / s\right)$. The headway is $H$ downtown, so the distance traveled per hour downtown $V_{d}$ is $\left(\left(4 D^{2} \alpha^{2}\right) /(s H)\right)$. The headway is $n H$ in the periphery, and the length of distance traveled is $2\left[2\left(D^{2} / s\right)(1-\alpha)^{2}\right]=\left(\left(4 D^{2}\left(1-\alpha^{2}\right)\right) / s\right)$. Thus, the total distance traveled per hour in the periphery is $V_{p}=\left(\left(4 D^{2}\left(1-\alpha^{2}\right)\right) /(\operatorname{sn} H)\right)$.

$V=V_{d}+V_{p}=\left(\left(4 D^{2}\left(\alpha^{2} n-\alpha^{2}+1\right)\right) /(H n s)\right)$.

Result 3. The expected number of transfers per trip is given by

$$
e_{T}=P_{1}=1-\frac{9 \alpha^{2} f s(D+\alpha D+f D-2 f s-\alpha f D)-3 s\left(D-s+\alpha D+f D-f s-\alpha^{2} f D\right)\left(2 \alpha+f-2 \alpha f-5 \alpha^{2} f+2 \alpha^{2}+2\right)}{2 D^{2}\left(2 \alpha+f-2 \alpha f-2 \alpha^{2} f+2 \alpha^{2}+2\right)^{2}},
$$

where $e_{T}$ is equal to the probability of only doing one transfer.

Zero transfer: for a passenger, zero transfer occurred when both the origin and destination fell in the service area of one bus line. The probability may be calculated by the ratio of the travel demand satisfying that condition to the total travel demands. Thus, the origins in downtown areas or in the periphery are treated differently. Downtown, the following is true:

$$
P_{0, d}=\frac{\int_{0}^{\alpha D} \int_{0}^{\alpha D} \mathrm{~d} x \mathrm{~d} y}{\int_{0}^{\alpha D} \int_{0}^{\alpha D} \mathrm{~d} x \mathrm{~d} y+2 \int_{\alpha D}^{D} \int_{0}^{x}(1-k x) \mathrm{d} x \mathrm{~d} y} \frac{\alpha s D-s^{2}+\int_{\alpha D}^{D} s(1-k x) \mathrm{d} x}{\int_{0}^{\alpha D} \int_{0}^{\alpha D} \mathrm{~d} x \mathrm{~d} y+2 \int_{\alpha D}^{D} \int_{0}^{x}(1-k x) \mathrm{d} x \mathrm{~d} y}
$$

In the periphery, the following is true:

$$
\begin{aligned}
P_{0, p} & =\frac{2 \int_{\alpha D}^{D} \int_{0}^{x}(1-k x) \mathrm{d} x \mathrm{~d} y}{\int_{0}^{\alpha D} \int_{0}^{\alpha D} \mathrm{~d} x \mathrm{~d} y+2 \int_{\alpha D}^{D} \int_{0}^{x}(1-k x) \mathrm{d} x \mathrm{~d} y} \frac{\alpha s D+\int_{\alpha D}^{D} s(1-k x) \mathrm{d} x-\int_{(((1-\alpha) D) / 2)-(s / 2)}^{(((1-\alpha) D) / 2)+(s / 2)} s(1-k x) \mathrm{d} x}{\int_{0}^{\alpha D} \int_{0}^{\alpha D} \mathrm{~d} x \mathrm{~d} y+2 \int_{\alpha D}^{D} \int_{0}^{x}(1-k x) \mathrm{d} x \mathrm{~d} y} \\
P_{0} & =P_{0, d}+P_{0, p}
\end{aligned}
$$

One transfer: the probability of one transfer is one subtracting the probability of zero transfer. From this, the following is obtained: $e_{T}=P_{1}=1-P_{0}$.

Result 4. The expected access and egress time are given by $A=\left(s / v_{w}\right)$.
The expected travel distance for one passenger at each stop was $s / 2$. Thus, the expected access and egress distance is equal to $s$.

Result 5. The expected waiting time per passenger, including the origin and transfer stops, is 


$$
\begin{aligned}
W= & \frac{H\left(2 n+2 \alpha n+f n+3 \alpha^{2} f+2 \alpha^{2} n-2 \alpha f n-5 \alpha^{2} f n\right)}{2\left(2 \alpha+f-2 \alpha f-2 \alpha^{2} f+2 \alpha^{2}+2\right)} \\
& \times\left[2-\frac{3 s\left(2 \alpha+f-2 \alpha f+5 \alpha^{2} f+2 \alpha^{2}+2\right)(D-s+\alpha D+f D-f s-\alpha f D)}{2 D^{2}\left(2 \alpha+f-2 \alpha f-2 \alpha^{2} f+2 \alpha^{2}+2\right)^{2}}-\frac{9 \alpha^{2} f s(D+\alpha D+f D-2 f s-\alpha f D)}{2 D^{2}\left(2 \alpha+f-2 \alpha f-2 \alpha^{2} f+2 \alpha^{2}+2\right)^{2}}\right]
\end{aligned}
$$

Proof. Assume that passengers arrive independently of the timetable. The expected waiting time has two components: (i) $W_{O}$, at the origin stop, and (ii) $W_{T}$, at the transfer stop. $W_{O}$ and $W_{T}$ were identical.

$W_{O}$ can be divided into waiting downtown $W_{O, d}$ and in the periphery $W_{O, p}$.

In downtown areas, the waiting time is $H / 2$ and, when multiplied by the probability of being located downtown, the following is obtained:

$$
W_{O, d}=\frac{H}{2} \cdot \frac{\int_{0}^{\alpha D} \mathrm{~d} x \int_{0}^{\alpha D} \mathrm{~d} y}{\int_{0}^{\alpha D} \int_{0}^{\alpha D} \mathrm{~d} x \mathrm{~d} y+2 \int_{\alpha D}^{D} \int_{0}^{x}(1-k x) \mathrm{d} x \mathrm{~d} y} .
$$

In the periphery, the waiting time is $n H / 2$. Multiplied by the probability of being located in the periphery, the following is obtained:

$$
W_{O, p}=\frac{n H}{2} \cdot \frac{2 \int_{\alpha D}^{D} \int_{0}^{x}(1-k x) \mathrm{d} x \mathrm{~d} y}{\int_{0}^{\alpha D} \int_{0}^{\alpha D} \mathrm{~d} x \mathrm{~d} y+2 \int_{\alpha D}^{D} \int_{0}^{x}(1-k x) \mathrm{d} x \mathrm{~d} y} .
$$

Thus, $W_{O}=W_{O, d}+W_{O, p}$ and $W=W_{O}+P_{1} \cdot W_{O}$.

Result 6. The expected in-vehicle travel distance per trip is given by

$$
\begin{aligned}
E= & \frac{3 D\left(50 \alpha^{5} f^{2}-100 \alpha^{5} f+50 \alpha^{5}-124 \alpha^{4} f^{2}+24 \alpha^{4} f+100 \alpha^{4}+129 \alpha^{3} f^{2}-167 \alpha^{3} f+38 \alpha^{3}-3 \alpha^{2} f^{2}-183 \alpha^{2} f\right)}{70\left(2 \alpha+f-2 \alpha f-2 \alpha^{2} f+2 \alpha^{2}+2\right)^{2}} \\
& +\frac{3 D\left(186 \alpha^{2}-65 \alpha f^{2}-59 \alpha f+124 \alpha+13 f^{2}+65 f+62\right)}{70\left(2 \alpha+f-2 \alpha f-2 \alpha^{2} f+2 \alpha^{2}+2\right)^{2}}
\end{aligned}
$$

Proof. According to the origin and destination location, travels can be divided into 3 cases: (a) both stops fall inside downtown; (b) both stops fall outside downtown; and (c) the rest. Let an $X Y$ coordinate system have as the origin the southwest corner of the city. Let the coordinates of the origin be $\left(x_{1}, y_{1}\right)$ and the coordinates of the destination be $\left(x_{2}, y_{2}\right)$.

For case (a), the travel distance between two random stops in downtown is $(2 / 3) \alpha D$ and the probability is

$$
\left(\frac{\int_{0}^{\alpha D} \int_{0}^{\alpha D} \mathrm{~d} x \mathrm{~d} y}{\int_{0}^{\alpha D} \int_{0}^{\alpha D} \mathrm{~d} x \mathrm{~d} y+2 \int_{\alpha D}^{D} \int_{0}^{x}(1-k x) \mathrm{d} x \mathrm{~d} y}\right)^{2} .
$$

Thus,

$$
E_{a}=\frac{2}{3} \alpha D\left(\frac{\int_{0}^{\alpha D} \int_{0}^{\alpha D} \mathrm{~d} x \mathrm{~d} y}{\int_{0}^{\alpha D} \int_{0}^{\alpha D} \mathrm{~d} x \mathrm{~d} y+2 \int_{\alpha D}^{D} \int_{0}^{x}(1-k x) \mathrm{d} x \mathrm{~d} y}\right)^{2} .
$$

For case (b), $\quad E_{b}=\left(\left|x_{1}-x_{2}\right|+\left|y_{1}-y_{2}\right|\right)$ $p\left(x_{1}\right) p\left(x_{2}\right) p\left(y_{1}\right) p\left(y_{2}\right)=4\left(x_{1}-x_{2}\right) p\left(x_{1}\right) p\left(x_{2}\right)\left(x_{1}>x_{2}\right)$, according to the symmetry of the coordinate system. Case (b) includes three scenarios: (1) the origin and destination fall in the eastern periphery; (2) the origin and destination 
fall in the northern periphery, as shown in Figures 4(a)-4(c); and (3) the rest, which is shown in Figures 4(d)-4(f).

$$
\begin{aligned}
& E_{b_{1}}=\frac{\int_{\alpha D}^{D} \int_{\alpha D}^{x_{1}}\left(x_{1}-x_{2}\right)\left(1-k x_{2}\right) x_{2}\left(1-k x_{1}\right) x_{1} \mathrm{~d} x_{2} \mathrm{~d} x_{1}}{\left(\int_{0}^{\alpha D} \int_{0}^{\alpha D} \mathrm{~d} x \mathrm{~d} y+2 \int_{\alpha D}^{D} \int_{0}^{x}(1-k x) \mathrm{d} x \mathrm{~d} y\right)^{2}}, \\
& E_{b_{2}}=\frac{\int_{0}^{\alpha D} \int_{\alpha D}^{D} \int_{0}^{x_{1}} \int_{\alpha D}^{D}\left(x_{1}-x_{2}\right)\left(1-k y_{2}\right) \mathrm{d} y_{2} \mathrm{~d} x_{2}\left(1-k y_{1}\right) \mathrm{d} y_{1} \mathrm{~d} x_{1}}{\left(\int_{0}^{\alpha D} \int_{0}^{\alpha D} \mathrm{~d} x \mathrm{~d} y+2 \int_{\alpha D}^{D} \int_{0}^{x}(1-k x) \mathrm{d} x \mathrm{~d} y\right)^{2}} \\
& +\frac{\int_{\alpha D}^{D} \int_{x_{1}}^{D} \int_{0}^{\alpha D} \int_{\alpha D}^{D}\left(x_{1}-x_{2}\right)\left(1-k y_{2}\right) \mathrm{d} y_{2} \mathrm{~d} x_{2}\left(1-k y_{1}\right) \mathrm{d} y_{1} \mathrm{~d} x_{1}}{\left(\int_{0}^{\alpha D} \int_{0}^{\alpha D} \mathrm{~d} x \mathrm{~d} y+2 \int_{\alpha D}^{D} \int_{0}^{x}(1-k x) \mathrm{d} x \mathrm{~d} y\right)^{2}} \\
& +\frac{\int_{\alpha D}^{D} \int_{x_{1}}^{D} \int_{\alpha D}^{x_{1}} \int_{x_{2}}^{D}\left(x_{1}-x_{2}\right)\left(1-k y_{2}\right)\left(1-k y_{1}\right) \mathrm{d} y_{2} \mathrm{~d} x_{2} \mathrm{~d} y_{1} \mathrm{~d} x_{1}}{\left(\int_{0}^{\alpha D} \int_{0}^{\alpha D} \mathrm{~d} x \mathrm{~d} y+2 \int_{\alpha D}^{D} \int_{0}^{x}(1-k x) \mathrm{d} x \mathrm{~d} y\right)^{2}}, \\
& E_{b_{3}}=\frac{\int_{\alpha D}^{D} \int_{\alpha D}^{D} \int_{0}^{\alpha D}\left(x_{1}-x_{2}\right)\left(1-k y_{2}\right) \mathrm{d} x_{2} \mathrm{~d} y_{2}\left(1-k x_{1}\right) x_{1} \mathrm{~d} x_{1}}{\left(\int_{0}^{\alpha D} \int_{0}^{\alpha D} \mathrm{~d} x \mathrm{~d} y+2 \int_{\alpha D}^{D} \int_{0}^{x}(1-k x) \mathrm{d} x \mathrm{~d} y\right)^{2}} \\
& +\frac{\int_{\alpha D}^{D} \int_{\alpha D}^{x_{1}} \int_{x_{2}}^{D}\left(x_{1}-x_{2}\right)\left(1-k y_{2}\right) \mathrm{d} y_{2} \mathrm{~d} x_{2}\left(1-k x_{1}\right) x_{1} \mathrm{~d} x_{1}}{\left(\int_{0}^{\alpha D} \int_{0}^{\alpha D} \mathrm{~d} x \mathrm{~d} y+2 \int_{\alpha D}^{D} \int_{0}^{x}(1-k x) \mathrm{d} x \mathrm{~d} y\right)^{2}} \\
& \cdot \frac{\int_{\alpha D}^{D} \int_{x 1}^{D} \int_{\alpha D}^{x_{1}}\left(x_{1}-x_{2}\right)\left(1-k x_{2}\right) x_{2} \mathrm{~d} x_{2}\left(1-k y_{1}\right) \mathrm{d} y_{1} \mathrm{~d} x_{1}}{\left(\int_{0}^{\alpha D} \int_{0}^{\alpha D} \mathrm{~d} x \mathrm{~d} y+2 \int_{\alpha D}^{D} \int_{0}^{x}(1-k x) \mathrm{d} x \mathrm{~d} y\right)^{2}}, \\
& E_{b}=4\left(E_{b_{1}}+E_{b_{2}}+E_{b_{3}}\right) \text {. }
\end{aligned}
$$

For case (c), $\quad E_{c}=\left(\left|x_{1}-x_{2}\right|+\left|y_{1}-y_{2}\right|\right) p$ $\left(x_{1}\right) p\left(x_{2}\right) p\left(y_{1}\right) p\left(y_{2}\right)=2\left|x_{1}-x_{2}\right| p\left(x_{1}\right) p\left(x_{2}\right)$, according to the symmetry of the coordinate system. Let the origin be inside downtown and the destination be in the periphery, as shown in Figure 5, or vice versa.

$$
\begin{aligned}
& E_{c}=4\left[\begin{array}{l}
\frac{\int_{0}^{\alpha D} \int_{0}^{x 1} \int_{\alpha D}^{D} \alpha D\left(x_{1}-x_{2}\right)\left(1-k y_{2}\right) \mathrm{d} y_{2} \mathrm{~d} x_{2} \mathrm{~d} x_{1}}{\left(\int_{0}^{\alpha D} \int_{0}^{\alpha D} \mathrm{~d} x \mathrm{~d} y+2 \int_{\alpha D}^{D} \int_{0}^{x}(1-k x) \mathrm{d} x \mathrm{~d} y\right)^{2}}+\frac{\int_{0}^{\alpha D} \int_{\alpha D}^{D} \int_{0}^{x 2} \alpha D\left(x_{2}-x_{1}\right) \mathrm{d} x_{1}\left(1-k y_{2}\right) \mathrm{d} y_{2} \mathrm{~d} x_{2}}{\left(\int_{0}^{\alpha D} \int_{0}^{\alpha D} \mathrm{~d} x \mathrm{~d} y+2 \int_{\alpha D}^{D} \int_{0}^{x}(1-k x) \mathrm{d} x \mathrm{~d} y\right)^{2}} \\
\frac{\int_{\alpha D}^{D} \int_{x 2}^{D} \int_{0}^{\alpha D} \alpha D\left(x_{2}-x_{1}\right) \mathrm{d} x 1\left(1-k y_{2}\right) \mathrm{d} y_{2} \mathrm{~d} x_{2}}{\left(\int_{0}^{\alpha D} \int_{0}^{\alpha D} \mathrm{~d} x \mathrm{~d} y+2 \int_{\alpha D}^{D} \int_{0}^{x}(1-k x) \mathrm{d} x \mathrm{~d} y\right)^{2}}+\frac{\int_{\alpha D}^{D} \int_{0}^{\alpha D} \alpha D\left(x_{2}-x_{1}\right) \mathrm{d} x_{1}\left(1-k x_{2}\right) x_{2} \mathrm{~d} x_{2}}{\left(\int_{0}^{\alpha D} \int_{0}^{\alpha D} \mathrm{~d} x \mathrm{~d} y+2 \int_{\alpha D}^{D} \int_{0}^{x}(1-k x) \mathrm{d} x \mathrm{~d} y\right)^{2}}
\end{array}\right], \\
& E=E_{a}+E_{b}+E_{c} .
\end{aligned}
$$

Result 7. The expected travel speed during the peak hour is given by

$$
\frac{1}{v_{c}} \approx \frac{1}{v}+\frac{\tau}{s}-\tau^{\prime} \frac{\Lambda\left(1+e_{T}\right)}{V}
$$

Result 8. The maximum occupancy on the critical load line during peak hours is approximately given by

$$
O=\frac{2 s H \Lambda}{\alpha D}\left(\frac{1}{2}-\frac{\alpha^{4}(2 \alpha+3 f-5 \alpha f)^{2}}{8(1-\alpha)^{2}\left(2 \alpha+f-2 \alpha f-2 \alpha^{2} f+2 \alpha^{2}+2\right)^{2}}\right) .
$$

Proof. This proof can be found in Daganzo's work [12]. 
Proof. These results can be seen by calculating the average passenger trips on the critical lines during peak hours and then multiplying the load factor by 2 . Passengers traveling across the equatorial lines are the most. For the purpose of this proof, passengers traveling from north to south are taken as an example. The number of passengers traveling this way is $(\Lambda / 4)$. Passengers traveling by east-west equatorial lines can be divided into two parts: (i) trips based on the peripheral lines and (ii) the rest. The number of trips belonging to (i) was

$$
\Lambda\left[\frac{\int_{\alpha D}^{D} \int_{0}^{x}(1-k x) \mathrm{d} x \mathrm{~d} y}{2\left(\int_{0}^{\alpha D} \int_{0}^{\alpha D} \mathrm{~d} x \mathrm{~d} y+2 \int_{\alpha D}^{D} \int_{0}^{x}(1-k x) \mathrm{d} x \mathrm{~d} y\right)}\right] .
$$

Therefore, the average number of trips crossing the equatorial lines in downtown areas is

$$
\frac{\Lambda}{4}-\Lambda\left[\frac{\int_{\alpha D}^{D} \int_{0}^{x}(1-k x) \mathrm{d} x \mathrm{~d} y}{2\left(\int_{0}^{\alpha D} \int_{0}^{\alpha D} \mathrm{~d} x \mathrm{~d} y+2 \int_{\alpha D}^{D} \int_{0}^{x}(1-k x) \mathrm{d} x \mathrm{~d} y\right)}\right]
$$

Because there are $\alpha \mathrm{D} / 2$ s critical lines and the headway is $\mathrm{H}$, the total number of vehicles per hour on these lines is $((\alpha D) /(2 s H))$. On each equatorial line, an average of

$$
\frac{\left\{(\Lambda / 4)-\Lambda\left[\left(\int_{\alpha D}^{D} \int_{0}^{x}(1-k x) \mathrm{d} x \mathrm{~d} y\right) /\left(2\left(\int_{0}^{\alpha D} \int_{0}^{\alpha D} \mathrm{~d} x \mathrm{~d} y+2 \int_{\alpha D}^{D} \int_{0}^{x}(1-k x) \mathrm{d} x \mathrm{~d} y\right)\right)\right]^{2}\right\}}{(\alpha D) /(2 s H)}
$$

passengers are traveling.

\section{Data Availability}

The [DATA TYPE] data used to support the findings of this study are included within the article.

\section{Conflicts of Interest}

The authors declare that there are no conflicts of interest regarding the publication of this paper.

\section{Acknowledgments}

This work was supported by the National Natural Science Foundation of China (Grant no. 51668048), the Xi'an Special Funds for Urban Construction Program (Grant no. SZJJ2019-22), and the Natural Science Foundation of Inner Mongolia Autonomous Region (Grant no. 2020MS05060).

\section{References}

[1] J. Walker, Human Transit, Island Press/Center for Resource Economics, Washington, DC, USA, 2012.

[2] S. B. Jha, J. K. Jha, and M. K. Tiwari, "A multi-objective metaheuristic approach for transit network design and frequency setting problem in a bus transit system," Computers \& Industrial Engineering, vol. 130, no. 4, pp. 166-186, 2019.

[3] E. Cipriani, G. Fusco, S. M. Patella, M. Petrelli, and L. Quadrifoglio, "Transit network design for small-medium size cities," Transportation Planning and Technology, vol. 42, no. 1, pp. 84-97, 2019.

[4] X. Feng, X. Zhu, X. Qian, Y. Jie, F. Ma, and X. Niu, "A new transit network design study in consideration of transfer time composition," Transportation Research Part D: Transport and Environment, vol. 66, pp. 85-94, 2019.
[5] D. Zhu, Y. Gu, S. Wang, Z. Liu, and W. Zhang, "A two-phase optimization model for the demand-responsive customized bus network design," Transportation Research Part C: Emerging Technologies, vol. 111, pp. 1-21, 2020.

[6] N. Oliker and S. Bekhor, "An infeasible start heuristic for the transit route network design problem," Transportmetrica A: Transport Science, vol. 16, no. 3, pp. 388-408, 2020.

[7] M. Nikolić and D. A. Teodorović, "Transit network design by bee colony optimization," Expert Systems with Applications, vol. 40, no. 15, pp. 5945-5955, 2013.

[8] D. Huang, Z. Liu, X. Fu, and P. T. Blythe, "Multimodal transit network design in a hub-and-spoke network framework," Transportmetrica A: Transport Science, vol. 14, no. 8, pp. 706-735, 2018.

[9] F. Zhao and I. Ubaka, "Transit network optimizationminimizing transfers and optimizing route directness," Journal of Public Transportation, vol. 7, no. 7, pp. 67-82, 2004.

[10] F. Zhao and X. Zeng, "Simulated annealing-genetic algorithm for transit network optimization," Journal of Computing in Civil Engineering, vol. 20, no. 1, pp. 57-68, 2006.

[11] J. Boissière, F. Martin, N. Teypaz et al., "Using choquetintegral for guiding tabu search in multi-criteria public transport network design," IFAC Proceedings, Volumes, vol. 40, no. 18, pp. 617-622, 2007.

[12] C. F. Daganzo, "Structure of competitive transit networks," Transportation Research Part B: Methodological, vol. 44, no. 4, pp. 434-446, 2010.

[13] M. Estrada, M. Roca-Riu, H. Badia, F. Robusté, and C. F. Daganzo, "Design and implementation of efficient transit networks: procedure, case study and validity test," Transportation Research Part A: Policy and Practice, vol. 45, no. 9, pp. 935-950, 2011.

[14] H. Badia, A. C. Juan, and C. F. Daganzo, "How network structure can boost and shape the demand for bus transit," Transportation Research Part A: Policy and Practice, vol. 103, no. 9, pp. 83-94, 2017.

[15] H. Badia, M. Estrada, and F. Robusté, "Competitive transit network design in cities with radial street patterns," 
Transportation Research Part B: Methodological, vol. 59, pp. 161-181, 2014.

[16] S. Nocera, A. Fabio, F. Cavallaro et al., "The adoption of grid transit networks in non-metropolitan contexts," Transportation Research Part A: Policy and Practice, vol. 132, no. 2, pp. 256-272, 2020.

[17] G. Chen and J. H. Wang, "Research on radial and grid hybrid bus network model for square cities of downtown in the corner," Journal of Transportation Systems Engineering and Information Technology, vol. 21, pp. 1-8, 2021, accepted.

[18] G. L. Thompson, "Planning considerations for alternative transit route structures," Journal of the American Institute of Planners, vol. 43, no. 2, pp. 158-168, 1977.

[19] B. F. Byrne, "Public transportation line positions and headways for minimum user and system cost in a radial case," Transportation Research, vol. 9, no. 2-3, pp. 97-102, 1975.

[20] G. F. Newell, "Some issues relating to the optimal design of bus routes," Transportation Science, vol. 13, no. 1, pp. 20-35, 1979.

[21] R. Vaughan, "Optimum polar networks for an urban bus system with a many-to-many travel demand," Transportation Research Part B: Methodological, vol. 20, no. 3, pp. 215-224, 1986.

[22] H. Badia, M. Estrada, and F. Robusté, "Bus network structure and mobility pattern: a monocentric analytical approach on a grid street layout," Transportation Research Part B: Methodological, vol. 93, no. 11, pp. 37-56, 2016.

[23] H. Pan and Y. Xiaoguang, "Probability distribution of per capita travel speed in urban road," Journal of Transportation Systems Engineering and Information Technology, vol. 16, no. 5, pp. 149-156, 2016.

[24] J. Zhang, "A real-time bus transfer scheme recommendation systems," in Proceedings of the International Conference on Advanced Cloud and Big Data, pp. 206-212, Shanghai, China, August 2017.

[25] D. Huang, Z. Liu, P. Liu, and J. Chen, "Optimal transit fare and service frequency of a nonlinear origin-destination based fare structure," Transportation Research Part E: Logistics and Transportation Review, vol. 96, no. 12, pp. 1-19, 2016.

[26] SFMTA Municipal Transportation Agency, SFMTA Ar18 Final Online 2, SFMTA, San Francisco, CA, USA, 2018.

[27] SFMTA Municipal Transportation Agency, Commuter Shuttle Program Annual Status Report, SFMTA, San Francisco, CA, USA, 2018.

[28] J. Zhao, J. Yu, X. Xia, J. Ye, and Y. Yuan, "Exclusive bus lane network design: a perspective from intersection operational dynamics," Networks and Spatial Economics, vol. 19, no. 4, pp. 1143-1171, 2019.

[29] Transportation Research Board, Transit Capacity and Quality of Service Manual, Transportation Research Board, Washington, DC, USA, 3rd edition, 2013.

[30] O. Heddebaut, B. Finn, S. Rabuel, and F. Rambaud, "The European bus with a high level of service (BHLS): concept and practice," Built Environment, vol. 36, no. 6, pp. 307-316, 2010.

[31] B. Finn, "Buses with high level of service: fundamental characteristics and recommendations for decision making and research," Cost action TU0603, Final report, European Cooperation in Science and Technology, Brussels, Belgium, 2011.

[32] K. An, "Battery electric bus infrastructure planning under demand uncertainty," Transportation Research Part C: Emerging Technologies, vol. 111, pp. 572-587, 2020. 